\title{
Benzotriazolyl-mediated 1,2-Shifts of
}

\section{Electron-rich Heterocycles.}

Alan R. Katritzky, * Sergey Bobrov, Niveen Khashab and Kostyantyn Kirichenko

Center for Heterocyclic Compounds, University of Florida, Department of Chemistry,

Gainesville, Florida 32611-7200, USA

e-mail: Katritzky@chem.ufl.edu

$\underline{\text { Table of Contents }}$

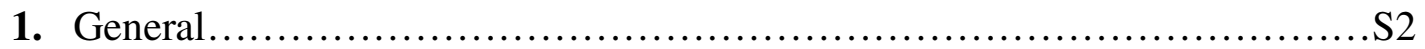

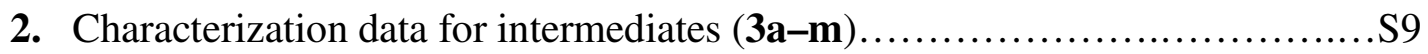

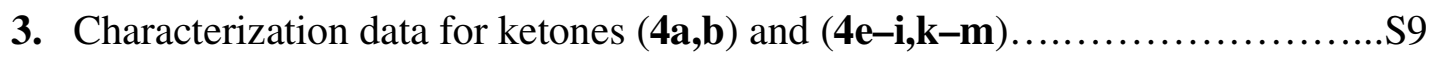


General. Melting points were determined on a hot-stage apparatus and are uncorrected. NMR spectra were recorded in $\mathrm{CDCl}_{3}$, acetone- $d_{6}$ or DMSO- $d_{6}$ as solvents with TMS as the internal standard for ${ }^{1} \mathrm{H}(300 \mathrm{MHz})$ or a solvent as the internal standard for ${ }^{13} \mathrm{C}(75$ MHz). THF was dried over sodium / benzophenone and used freshly distilled. Column chromatography was conducted on silica gel 200-425 meshes.

1-(1H-1,2,3-Benzotriazol-1-yl)-1-(methylthio)-2-(2-thienyl)-2-propanol (3a) (one diastereomer): microcrystals from acetone-diethyl ether (41\%); mp $138-139{ }^{\circ} \mathrm{C} ;{ }^{1} \mathrm{H}$ $\operatorname{NMR}\left(\mathrm{CDCl}_{3}\right): \delta 7.95(\mathrm{~d}, J=8.2 \mathrm{~Hz}, 1 \mathrm{H}), 7.73(\mathrm{~d}, J=8.3 \mathrm{~Hz}, 1 \mathrm{H}), 7.45-7.40(\mathrm{~m}, 1 \mathrm{H})$, 7.35-7.29 (m, 1H), $7.00(\mathrm{dd}, J=4.7,1.4 \mathrm{~Hz}, 1 \mathrm{H}), 6.76-6.68(\mathrm{~m}, 2 \mathrm{H}), 6.03(\mathrm{~s}, 1 \mathrm{H}), 4.09$ (s, 1H), $2.02(\mathrm{~s}, 3 \mathrm{H}), 1.92(\mathrm{~s}, 3 \mathrm{H}) ;{ }^{13} \mathrm{C} \mathrm{NMR}\left(\mathrm{CDCl}_{3}\right): \delta 148.3,145.6,132.8,127.3,126.7$, $124.8,124.1,123.9,119.7,111.9,77.4,75.7,29.0,14.7$. (Mixture of two diastereomers): colorless oil (82\%); ${ }^{1} \mathrm{H} \mathrm{NMR}\left(\mathrm{CDCl}_{3}\right): \delta 8.06(\mathrm{~d}, J=8.1 \mathrm{~Hz}, 1 \mathrm{H}), 7.97$ (d, $J=8.2 \mathrm{~Hz}, 1 \mathrm{H}), 7.85(\mathrm{~d}, J=8.2 \mathrm{~Hz}, 1 \mathrm{H}), 7.47-7.30(\mathrm{~m}, 4 \mathrm{H}), 7.28-7.25(\mathrm{~m}, 1 \mathrm{H}), 7.01$ (dd, $J=4.6,1.6 \mathrm{~Hz}, 1 \mathrm{H}), 6.97-6.95(\mathrm{~m}, 2 \mathrm{H}), 6.73-6.68(\mathrm{~m}, 2 \mathrm{H}), 6.07(\mathrm{~s}, 1 \mathrm{H}), 6.02(\mathrm{~s}$, 1H), $3.88(\mathrm{~s}, 1 \mathrm{H}), 3.29(\mathrm{~s}, 1 \mathrm{H}), 2.02(\mathrm{~s}, 3 \mathrm{H}), 1.94(\mathrm{~s}, 3 \mathrm{H}), 1.84(\mathrm{~s}, 3 \mathrm{H}), 1.57(\mathrm{~s}, 1 \mathrm{H})$, (double set of signals); ${ }^{13} \mathrm{C}$ NMR $\left(\mathrm{CDCl}_{3}\right): \delta 148.8,148.3,146.2,145.6,132.8,132.5$, $127.3,127.2,127.1,126.7,124.9,124.8,124.2,124.1,123.9,123.8,119.7,119.7,112.9$, 112.1, 77.3, 77.3, 77.1, 75.8, 29.0, 28.8, 14.9, 14.7, (double set of signals). Anal. Calcd for $\mathrm{C}_{14} \mathrm{H}_{15} \mathrm{~N}_{3} \mathrm{OS}_{2}$ : C, 55.06; H, 4.95; N, 13.76. Found: C, 55.25; H, 5.14; N, 13.85.

\section{1-(1H-1,2,3-Benzotriazol-1-yl)-1-(methylthio)-2-(3-thienyl)-2-propanol}

(mixture of two diastereomers): microcrystals from diethyl ether (88 \%), mp 118-122 ${ }^{\circ} \mathrm{C} ;{ }^{1} \mathrm{H}$ NMR $\left(\mathrm{CDCl}_{3}\right): \delta 8.03(\mathrm{~d}, J=8.2 \mathrm{~Hz}, 1 \mathrm{H}), 7.93(\mathrm{~d}, J=8.2 \mathrm{~Hz}, 1 \mathrm{H}), 7.83(\mathrm{~d}, J=$ 
$8.1 \mathrm{~Hz}, 1 \mathrm{H}), 7.70(\mathrm{~d}, J=8.2 \mathrm{~Hz}, 1 \mathrm{H}), 7.45-7.35$ (m, 3H), 7.33-7.26 (m, 3H), 7.10 (dd, $J$ $=4.7,1.7 \mathrm{~Hz}, 1 \mathrm{H}), 7.05(\mathrm{dd}, J=5.0,3.0 \mathrm{~Hz}, 1 \mathrm{H}), 6.91(\mathrm{dd}, J=3.0,1.4 \mathrm{~Hz}, 1 \mathrm{H}), 6.83(\mathrm{dd}$, $J=5.1,1.4 \mathrm{~Hz}, 1 \mathrm{H}), 6.07(\mathrm{~s}, 1 \mathrm{H}), 6.04(\mathrm{~s}, 1 \mathrm{H}), 3.74(\mathrm{~s}, 1 \mathrm{H}), 3.30(\mathrm{~s}, 1 \mathrm{H}), 1.93(\mathrm{~s}, 3 \mathrm{H})$, $1.91(\mathrm{~s}, 3 \mathrm{H}), 1.78(\mathrm{~s}, 3 \mathrm{H}), 1.50(\mathrm{~s}, 3 \mathrm{H}),\left(\right.$ double set of signals); ${ }^{13} \mathrm{C} \mathrm{NMR}\left(\mathrm{CDCl}_{3}\right): \delta$ $146.3,145.7,145.6,145.3,132.6,132.5,127.3,127.1,126.3,126.0,125.3,124.9,124.2$, 124.0, 121.6, 121.2, 119.8, 119.7, 112.8, 112.1, 76.9, 75.7, 28.2, 28.0, 14.7, 14.6, (double set of signals). Anal. Calcd for $\mathrm{C}_{14} \mathrm{H}_{15} \mathrm{~N}_{3} \mathrm{OS}_{2}$ : C, 55.06; H, 4.95; N, 13.76. Found: C, $55.35 ; \mathrm{H}, 5.03 ; \mathrm{N}, 13.80$.

\section{1-(1H-1,2,3-Benzotriazol-1-yl)-2-(1-methyl-1 $H$-indol-3-yl)-1-(methylthio)-2-}

propanol (3c) (one diastereomer): microcrystals from acetone-diethyl ether (41\%), mp $186-187{ }^{\circ} \mathrm{C} ;{ }^{1} \mathrm{H}$ NMR $\left(\mathrm{CDCl}_{3}\right): \delta$ 8.07-8.04 (m, 1H), 7.90-7.86 (m, 2H), 7.43-7.24 (m, 4H), 7.21-7.16 (m, 1H), $7.07(\mathrm{~s}, 1 \mathrm{H}), 6.42(\mathrm{~s}, 1 \mathrm{H}), 3.74(\mathrm{~s}, 3 \mathrm{H}), 3.11(\mathrm{~s}, 1 \mathrm{H}), 1.75(\mathrm{~s}$, $3 \mathrm{H}), 1.58(\mathrm{~s}, 3 \mathrm{H}) ;{ }^{13} \mathrm{C} \mathrm{NMR}\left(\mathrm{CDCl}_{3}\right): \delta 146.3,137.6,132.8,127.1,126.8,124.9,124.1$, $121.8,120.1,119.8,119.6,118.3,112.8,109.8,76.7,75.7,32.8,27.5,14.7$. (Mixture of two diastereomers): colorless oil $(82 \%)$; ${ }^{1} \mathrm{H}$ NMR $\left(\mathrm{CDCl}_{3}\right): \delta 8.06-8.03(\mathrm{~m}, 1 \mathrm{H}), 7.92-$ 7.86 (m, 3H), 7.78-7.74 (m, 1H), 7.56-7.53 (m, 1H), 7.42-7.32 (m, 3H), 7.31-7.23 (m, 4H), 7.22-7.15 (m, 3H), 7.10-7.04 (m, 2H), 6.78 (s, 1H), 6.40 (s, 1H), 6.35 (s, 1H), 3.72 (s, 3H), 3.64 (s, 1H), 3.53 (s, 3H), $3.13(\mathrm{~s}, 1 \mathrm{H}), 2.05$ (s, 3H), $1.86(\mathrm{~s}, 3 \mathrm{H}), 1.74(\mathrm{~s}, 3 \mathrm{H})$, $1.56(3 \mathrm{H})$, (double set of signals); ${ }^{13} \mathrm{C} \mathrm{NMR}\left(\mathrm{CDCl}_{3}\right): \delta 146.3,145.5,137.6,137.3,133.2$, $132.8,127.1,127.0,126.8,126.7,124.9,124.7,124.1,123.8,121.8,121.6,120.1,119.9$, 119.8, 119.6, 119.6, 119.3, 118.3, 118.0, 112.8, 111.6, 109.8, 109.6, 76.7, 75.7, 74.3, 32.8, 32.6, 27.5, 27.5, 14.7 (double set of signals). Anal. Calcd for $\mathrm{C}_{19} \mathrm{H}_{20} \mathrm{~N}_{4} \mathrm{OS}: \mathrm{C}$, 64.75; H, 5.72; N, 15.90. Found: C, 64.94; H, 5.84; N, 15.70. 
1-(1H-1,2,3-Benzotriazol-1-yl)-2-(2-furyl)-1-(methylthio)-2-propanol (3d) (first

diastereomer): microcrystals from acetone-diethyl ether $(37 \%), \mathrm{mp} 127-128{ }^{\circ} \mathrm{C} ;{ }^{1} \mathrm{H}$ $\operatorname{NMR}\left(\mathrm{CDCl}_{3}\right): \delta 7.98(\mathrm{~d}, J=8.2 \mathrm{~Hz}, 1 \mathrm{H}), 7.62(\mathrm{~d}, J=8.2 \mathrm{~Hz}, 1 \mathrm{H}), 7.45-7.39(\mathrm{~m}, 1 \mathrm{H})$, 7.35-7.29 (m, 1H), $7.13(\mathrm{dd}, J=1.8,0.8 \mathrm{~Hz}, 1 \mathrm{H}), 6.13(\mathrm{~s}, 1 \mathrm{H}), 6.08(\mathrm{dd}, J=3.4,1.8 \mathrm{~Hz}$, $1 \mathrm{H}), 6.03(\mathrm{dd}, J=3.4,0.8 \mathrm{~Hz}, 1 \mathrm{H}), 3.96(\mathrm{~s}, 1 \mathrm{H}), 1.96(\mathrm{~s}, 3 \mathrm{H}), 1.87(\mathrm{~s}, 3 \mathrm{H}) ;{ }^{13} \mathrm{C} \mathrm{NMR}$ $\left(\mathrm{CDCl}_{3}\right): \delta 155.5,145.6,141.9,132.8,127.3,124.1,119.8,111.4,110.4,106.7,75.3$ 73.7, 25.2, 14.7. Anal. Calcd for $\mathrm{C}_{14} \mathrm{H}_{15} \mathrm{~N}_{3} \mathrm{O}_{2} \mathrm{~S}$ : C, 58.11; H, 5.23; N, 14.52. Found: C, 58.25; H, 5.42; N, 14.63. (Second diastereomer): colorless oil (35\%); ${ }^{1} \mathrm{H} \mathrm{NMR}$ $\left(\mathrm{CDCl}_{3}\right): \delta 8.06(\mathrm{~d}, J=8.2 \mathrm{~Hz}, 1 \mathrm{H}), 7.91(\mathrm{~d}, J=8.3 \mathrm{~Hz}, 1 \mathrm{H}), 7.49-7.33(\mathrm{~m}, 3 \mathrm{H}), 6.40-$ $6.36(\mathrm{~m}, 2 \mathrm{H}), 6.25(\mathrm{~s}, 1 \mathrm{H}), 3.37(\mathrm{~s}, 1 \mathrm{H}), 1.84(\mathrm{~s}, 3 \mathrm{H}), 1.46(\mathrm{~s}, 3 \mathrm{H}) ;{ }^{13} \mathrm{C} \mathrm{NMR}\left(\mathrm{CDCl}_{3}\right): \delta$ $155.9,146.1,141.9,132.3,127.1,124.1,119.4,113.3,110.5,106.5,75.4,74.9,25.7$, 14.5 .

\section{2-(1-Benzofuran-2-yl)-1-(1H-1,2,3-benzotriazol-1-yl)-1-(methylthio)-2-propanol}

(3e) (mixture of two diastereomers): microcrystals from diethyl ether (75\%), mp 141-142 ${ }^{\circ} \mathrm{C} ;{ }^{1} \mathrm{H}$ NMR $\left(\mathrm{CDCl}_{3}\right): \delta 8.07-8.02(\mathrm{~m}, 2 \mathrm{H}), 7.90(\mathrm{~d}, J=8.3 \mathrm{~Hz}, 1 \mathrm{H}), 7.70(\mathrm{~d}, J$ $=8.3 \mathrm{~Hz}, 1 \mathrm{H}), 7.57-7.54(\mathrm{~m}, 1 \mathrm{H}), 7.49-7.20(\mathrm{~m}, 9 \mathrm{H}), 7.16-7.05(\mathrm{~m}, 2 \mathrm{H}), 6.85(\mathrm{~s}, 1 \mathrm{H})$, $6.46(\mathrm{~s}, 1 \mathrm{H}), 6.41(\mathrm{~s}, 1 \mathrm{H}), 6.27(\mathrm{~s}, 1 \mathrm{H}), 4.41(\mathrm{~s}, 1 \mathrm{H}), 3.89(\mathrm{~s}, 1 \mathrm{H}), 1.98(\mathrm{~s}, 3 \mathrm{H}), 1.96(\mathrm{~s}$, $3 \mathrm{H}), 1.84(\mathrm{~s}, 3 \mathrm{H}), 1.46(\mathrm{~s}, 3 \mathrm{H})$, (double set of signals); ${ }^{13} \mathrm{C}$ NMR (DMSO- $\left.d_{6}\right): \delta 160.4$, $160.0,154.2,154.1,146.0,145.5,132.4,132.2,127.9,127.5,126.9,126.6,124.1,124.1$, $124.0,123.8,122.9,122.7,121.1,121.0,119.1,118.9,114.3,114.3,111.2,110.9,103.3$, 102.9, 74.6, 74.5, 74.4, 25.6, 25.4, 14.0, 13.9, (double set of signals). Anal. Calcd for $\mathrm{C}_{18} \mathrm{H}_{17} \mathrm{~N}_{3} \mathrm{O}_{2} \mathrm{~S}: \mathrm{C}, 63.70 ; \mathrm{H}, 5.05 ; \mathrm{N}, 12.38$. Found: C, 63.68; H, 5.04; N, 12.36. 


\section{2-(1-Benzothiophen-2-yl)-1-(1H-1,2,3-benzotriazol-1-yl)-1-(methylthio)-2-}

propanol (3f) (mixture of two diastereomers): microcrystals from diethyl etheracetone (94\%), mp $139-140{ }^{\circ} \mathrm{C} ;{ }^{1} \mathrm{H}$ NMR $\left(\mathrm{CDCl}_{3}\right): \delta 8.06(\mathrm{~d}, J=8.4 \mathrm{~Hz}, 1 \mathrm{H}), 8.00(\mathrm{~d}, J$ $=8.3 \mathrm{~Hz}, 1 \mathrm{H}), 7.91(\mathrm{~d}, J=8.3 \mathrm{~Hz}, 1 \mathrm{H}), 7.83-7.70(\mathrm{~m}, 3 \mathrm{H}), 7.62-7.59(\mathrm{~m}, 1 \mathrm{H})$, 7.52-7.49 (m, 1H), 7.47-7.14 (m, 9H), $7.02(\mathrm{~s}, 1 \mathrm{H}), 6.17(\mathrm{~s}, 1 \mathrm{H}), 6.11(\mathrm{~s}, 1 \mathrm{H}), 4.37(\mathrm{~s}$, 1H), $3.64(\mathrm{~s}, 1 \mathrm{H}), 2.05(\mathrm{~s}, 3 \mathrm{H}), 1.94(\mathrm{~s}, 3 \mathrm{H}), 1.82(\mathrm{~s}, 3 \mathrm{H}), 1.5(\mathrm{~s}, 3 \mathrm{H})$, (double set of signals); ${ }^{13} \mathrm{C}$ NMR $\left(\mathrm{CDCl}_{3}\right): \delta 149.4,149.1,146.3,145.4,139.6,139.3,139.2,139.0$, $132.9,132.5,127.5,127.4,124.4,124.4,124.3,124.2,124.2,124.2,123.6,123.5,122.3$, 122.0, 120.7, 120.6, 119.8, 119.8, 113.0, 111.6, 77.7, 77.7, 76.7, 74.6, 29.1, 28.8, 14.9, 14.7, (double set of signals). Anal. Calcd for $\mathrm{C}_{18} \mathrm{H}_{17} \mathrm{~N}_{3} \mathrm{OS}_{2}$ : C, 60.82; H, 4.82; N, 11.82 . Found: C, 60.77; H, 4.74; N, 11.77.

\section{2-(1-Benzothiophen-3-yl)-1-(1H-1,2,3-benzotriazol-1-yl)-1-(methylthio)-2-}

propanol (3g) (first diastereomer): microcrystals from diethyl ether-acetone (20\%); mp $159-160{ }^{\circ} \mathrm{C} ;{ }^{1} \mathrm{H}$ NMR $\left(\mathrm{CDCl}_{3}\right): \delta 8.15(\mathrm{~d}, J=8.1 \mathrm{~Hz}, 1 \mathrm{H}), 7.90(\mathrm{~d}, J=8.1 \mathrm{~Hz}, 1 \mathrm{H}), 7.72$ $(\mathrm{d}, J=8.1 \mathrm{~Hz}, 1 \mathrm{H}), 7.57(\mathrm{~d}, J=8.1 \mathrm{~Hz}, 1 \mathrm{H}), 7.41-7.36(\mathrm{~m}, 1 \mathrm{H}), 7.33-7.24(\mathrm{~m}, 3 \mathrm{H}) 7.21$ $(\mathrm{s}, 1 \mathrm{H}), 6.47(\mathrm{~s}, 1 \mathrm{H}), 4.01(\mathrm{~s}, 1 \mathrm{H}), 2.12(\mathrm{~s}, 3 \mathrm{H}), 1.89(\mathrm{~s}, 3 \mathrm{H}) ;{ }^{13} \mathrm{C} \mathrm{NMR}\left(\mathrm{CDCl}_{3}\right): \delta$ 145.4, $141.3,138.6,135.7,132.9,127.3,124.0,124.0,123.2,123.1,119.9,111.0,78.1,73.1$, 26.8, 14.7. Anal. Calcd for $\mathrm{C}_{18} \mathrm{H}_{17} \mathrm{~N}_{3} \mathrm{OS}_{2}: \mathrm{C}, 60.82 ; \mathrm{H}, 4.82 ; \mathrm{N}, 11.82$. Found: $\mathrm{C}, 60.92 ; \mathrm{H}$, 4.78; N, 11.65. (Second diastereomer): colorless oil (20\%); $\delta 8.23(\mathrm{~d}, J=8.0 \mathrm{~Hz}, 1 \mathrm{H})$, $7.95(\mathrm{~d}, J=8.3 \mathrm{~Hz}, 1 \mathrm{H}), 7.88(\mathrm{~d}, J=8.2 \mathrm{~Hz}, 1 \mathrm{H}), 7.72(\mathrm{~d}, J=8.0 \mathrm{~Hz}, 1 \mathrm{H}), 7.41-7.33(\mathrm{~m}$, 2H), 7.30-7.25 (m, 2H), 7.23 (s, 1H), 6.49 (s, 1H), 5.41 (s, 1H), 2.09 (s, 3H), 1.80 (s, $3 \mathrm{H}) ;{ }^{13} \mathrm{C} \mathrm{NMR}\left(\mathrm{CDCl}_{3}\right): \delta 145.5,140.7,139.2,136.0,132.5,126.6,123.6,123.6,123.6$, 122.6, 119.1, 112.7, 77.1, 74.5, 26.5, 14.3. 


\section{2-(1H-1,2,3-Benzotriazol-1-yl)-2-(methylthio)-1-phenyl-1-(2-thienyl)-1-ethanol}

(3h) (mixture of two diastereomers): microcrystals from diethyl ether (75\%); mp $182-183{ }^{\circ} \mathrm{C} ;{ }^{1} \mathrm{H}$ NMR $\left(\mathrm{CDCl}_{3}\right): \delta 8.03(\mathrm{~d}, J=8.2 \mathrm{~Hz}, 1 \mathrm{H}), 7.93-7.83(\mathrm{~m}, 3 \mathrm{H}), 7.79-7.76$ (m, 2H), 7.54-7.26 (m, 11H), 7.05-6.95 (m, 5H), $6.73(\mathrm{dd}, J=3.7,1.1 \mathrm{~Hz}, 1 \mathrm{H}), 6.67(\mathrm{~s}$, 1H), $6.62(\mathrm{dd}, J=5.1,3.7 \mathrm{~Hz}, 1 \mathrm{H}), 6.56(\mathrm{~s}, 1 \mathrm{H}), 4.92(\mathrm{~s}, 1 \mathrm{H}), 4.57(\mathrm{~s}, 1 \mathrm{H}), 1.95(\mathrm{~s}, 3 \mathrm{H})$, $1.82(\mathrm{~s}, 3 \mathrm{H})$, (double set of signals); ${ }^{13} \mathrm{C}$ NMR (DMSO- $\left.d_{6}\right): \delta 149.9,149.1,145.7,145.4$, 144.0, 143.2, 132.7, 132.1, 128.0, 127.4, 127.2, 127.0, 126.7, 126.6, 126.2, 125.8, 125.4, $125.3,124.7,124.4,123.8,123.8,119.5,118.9,118.8,114.9,114.4,111.6,80.3,80.2$, 75.8, 75.5, 14.1, 14.0, (double set of signals). Anal. Calcd for $\mathrm{C}_{19} \mathrm{H}_{17} \mathrm{~N}_{3} \mathrm{OS}_{2}: \mathrm{C}, 62.10 ; \mathrm{H}$, 4.66; N, 11.43. Found: C, 62.35; H, 4.30; N, 11.33.

2-(1H-1,2,3-Benzotriazol-1-yl)-1-(2-furyl)-2-(methylthio)-1-phenyl-1-ethanol

(mixture of two diasterisomers): microcrystals from diethyl ether-acetone (40\%); mp 170-172 ${ }^{\circ} \mathrm{C} ;{ }^{1} \mathrm{H}$ NMR $\left(\mathrm{CDCl}_{3}\right): \delta 8.01(\mathrm{~d}, J=8.4 \mathrm{~Hz}, 1 \mathrm{H}), 7.95-7.88(\mathrm{~m}, 2 \mathrm{H}), 7.83-7.76$ (m, 3H), 7.50-7.28 (m, 10H), 7.03-6.99 (m, 4H), $6.74(\mathrm{~s}, 1 \mathrm{H}), 6.63(\mathrm{~d}, J=3.3, \mathrm{~Hz} 1 \mathrm{H})$, $6.56(\mathrm{~s}, 1 \mathrm{H}), 6.46(\mathrm{dd}, J=3.3,1.8 \mathrm{~Hz}, 1 \mathrm{H}), 6.14(\mathrm{~d}, J=3.3 \mathrm{~Hz}, 1 \mathrm{H}), 6.01(\mathrm{dd}, J=3.3,1.9$ $\mathrm{Hz}, 1 \mathrm{H}), 4.70(\mathrm{~s}, 1 \mathrm{H}), 4.32(\mathrm{~s}, 1 \mathrm{H}), 1.92(\mathrm{~s}, 3 \mathrm{H}), 1.81$ (s, 3H), (double sets of signals); ${ }^{13} \mathrm{C}$ NMR $\left(\mathrm{CDCl}_{3}\right): \delta 155.0,155.0,145.7,145.4,142.2,142.1,140.5,140.4,133.1,132.7$, $128.4,128.2,128.0,127.8,127.6,127.4,125.6,124.7,124.3,124.0,120.0,119.8,111.9$, 111.1, 110.9, 110.4, 107.5, 107.4, 79.7, 79.1, 73.1, 72.6, 14.7 (double sets of signals). Anal. Calcd for $\mathrm{C}_{19} \mathrm{H}_{17} \mathrm{~N}_{3} \mathrm{O}_{2} \mathrm{~S}: \mathrm{C}, 64.94 ; \mathrm{H}, 4.88 ; \mathrm{N}, 11.96$. Found: C, 65.05; H, 4.85; N, 12.01 .

2-(1H-1,2,3-Benzotriazol-1-yl)-1-(1-methyl-1H-indol-3-yl)-2-(methylthio)-1phenyl-1-ethanol (3j) (one diastereomer): microcrystals from diethyl ether (36\%); mp 
119-120 ${ }^{\circ} \mathrm{C} ;{ }^{1} \mathrm{H}$ NMR $\left(\mathrm{CDCl}_{3}\right): \delta 7.79(\mathrm{~d}, J=7.8 \mathrm{~Hz}, 1 \mathrm{H}), 7.82(\mathrm{~d}, J=8.1 \mathrm{~Hz}, 1 \mathrm{H})$, 7.67-7.64 (m, 2H), 7.41-7.25 (m, 7H), 7.15-7.04 (m, 2H), 6.88-6.82 (m, 1H), $6.69(\mathrm{~s}$, $1 \mathrm{H}), 3.64(\mathrm{~s}, 3 \mathrm{H}), 3.58(\mathrm{~s}, 1 \mathrm{H}), 1.72(\mathrm{~s}, 3 \mathrm{H}) ;{ }^{13} \mathrm{C} \mathrm{NMR}\left(\mathrm{CDCl}_{3}\right): \delta 146.1,143.0,137.0$, 133.0, 128.0, 127.6, 127.2, 127.0, 126.4, 125.7, 124.1, 121.9, 120.7, 119.7, 119.4, 117.1, 112.9, 109.3, 80.0, 75.3, 32.8, 14.7. (Mixture of two diastereomers): colorless oil (72\%); ${ }^{1} \mathrm{H}$ NMR (DMSO- $\left.d_{6}-\mathrm{CDCl}_{3}\right): \delta 8.35(\mathrm{~d}, J=8.4 \mathrm{~Hz}, 1 \mathrm{H}), 7.96-7.84(\mathrm{~m}, 2 \mathrm{H}), 7.83$ (d, $J=8.2 \mathrm{~Hz}, 1 \mathrm{H}), 7.70-7.67(\mathrm{~m}, 3 \mathrm{H}), 7.48-7.10(\mathrm{~m}, 15 \mathrm{H}), 7.03-6.98(\mathrm{~m}, 1 \mathrm{H}), 6.93-$ $6.85(\mathrm{~m}, 5 \mathrm{H}), 6.77-6.73(\mathrm{~m}, 2 \mathrm{H}), 5.81(\mathrm{~s}, 1 \mathrm{H}), 5.54(\mathrm{~s}, 1 \mathrm{H}), 3.86(\mathrm{~s}, 3 \mathrm{H}), 3.62(\mathrm{~s}, 3 \mathrm{H})$, $1.84(\mathrm{~s}, 3 \mathrm{H}), 1.70(\mathrm{~s}, 3 \mathrm{H}),\left(\right.$ double set of signals); ${ }^{13} \mathrm{C}$ NMR (DMSO- $\left.d_{6}-\mathrm{CDCl}_{3}\right): \delta 145.6$, $145.5,143.2,142.7,136.6,136.3,132.5,131.8,127.2,126.9,126.6,126.6,126.4,126.1$, $125.9,125.9,125.7,125.4,125.1,123.3,123.1,121.1,121.0,120.9,120.7,118.6,118.5$, $118.3,118.3,117.1,117.0,114.2,113.5,108.7,108.4,78.7,78.6,76.0,75.1,32.4,32.2$, 14.0, 13.7, (double set of signals). Anal. Calcd for $\mathrm{C}_{24} \mathrm{H}_{22} \mathrm{~N}_{4} \mathrm{OS}$ : C, 69.54; H, 5.35; N, 13.52. Found: C, 69.83; H, 5.49; N, 13.94 .

\section{1-(1-Benzofuran-2-yl)-2-(1H-1,2,3-benzotriazol-1-yl)-2-(methylthio)-1-phenyl-1-}

ethanol (3k) (mixture of two diastereomers): microcrystals from diethyl ether (70\%); mp 88-90 ${ }^{\circ} \mathrm{C} ;{ }^{1} \mathrm{H}$ NMR $\left(\mathrm{CDCl}_{3}\right): \delta 7.97-7.88(\mathrm{~m}, 5 \mathrm{H}), 7.82(\mathrm{~d}, J=8.4 \mathrm{~Hz}, 1 \mathrm{H})$, 7.61-7.59 (m, 1H), $7.54(\mathrm{~d}, J=8.1 \mathrm{~Hz}, 1 \mathrm{H}), 7.51-7.36(\mathrm{~m}, 7 \mathrm{H}), 7.34-7.27(\mathrm{~m}, 5 \mathrm{H}), 7.17$ (d, $J=8.1 \mathrm{~Hz}, 1 \mathrm{H}), 7.11-7.08(\mathrm{~m}, 1 \mathrm{H}), 7.05-6.98(\mathrm{~m}, 5 \mathrm{H}), 6.88(\mathrm{~s}, 1 \mathrm{H}), 6.71(\mathrm{~s}, 1 \mathrm{H})$, $6.64(\mathrm{~s}, 1 \mathrm{H}), 5.10(\mathrm{~s}, 1 \mathrm{H}), 4.68(\mathrm{~s}, 1 \mathrm{H}), 1.95(\mathrm{~s}, 3 \mathrm{H}), 1.85(\mathrm{~s}, 3 \mathrm{H})$, (double set of signals); ${ }^{13} \mathrm{C} \mathrm{NMR}\left(\mathrm{CDCl}_{3}\right): \delta 157.7,157.6,154.8,154.4,145.6,145.2,140.2,140.1,133.2,132.9$, $128.5,128.4,128.1,128.1,128.0,127.8,127.6,127.5,125.6,124.7,124.5,124.4,124.2$, 124.2, 123.2, 122.8, 121.5, 121.3, 120.1, 119.9, 111.5, 111.3, 110.8, 110.7, 104.3, 104.1, 
80.0, 79.3, 72.2, 71.8, 14.8, 14.8, (double set of signals). Anal. Calcd for $\mathrm{C}_{23} \mathrm{H}_{19} \mathrm{~N}_{3} \mathrm{O}_{2} \mathrm{~S}: \mathrm{C}$, 68.81; H, 4.77; N, 10.47. Found: C, 68.74; H, 4.79; N, 10.51.

1-(1-Benzothiophen-2-yl)-2-(1H-1,2,3-benzotriazol-1-yl)-2-(methylthio)-1-phenyl1-ethanol (3I) (mixture of two diastereomers): microcrystals from diethyl ether (98\%); mp $165-166{ }^{\circ} \mathrm{C} ;{ }^{1} \mathrm{H}$ NMR $\left(\mathrm{CDCl}_{3}\right): \delta 8.01-7.90(\mathrm{~m}, 3 \mathrm{H}), 7.84-7.75(\mathrm{~m}, 5 \mathrm{H}), 7.55-7.29$ (m, 15H), 7.19-7.13 (m, 2H), 7.09 (s, 1H), 7.05-6.97 (m, 2H), $6.77(\mathrm{~s}, 1 \mathrm{H}), 6.65(\mathrm{~s}, 1 \mathrm{H})$, $5.31(\mathrm{~s}, 1 \mathrm{H}), 4.83(\mathrm{~s}, 1 \mathrm{H}), 1.97(\mathrm{~s}, 3 \mathrm{H}), 1.84(\mathrm{~s}, 3 \mathrm{H})$, (double set of signals); ${ }^{13} \mathrm{C}$ NMR $\left(\mathrm{CDCl}_{3}\right): \delta 149.1,148.7,145.5,145.4,141.9,141.7,139.6,139.3,139.3,139.0,133.3$, 132.7, $128.45128 .2,128.0,128.0,127.8,127.7,125.5,124.7,124.6,124.5,124.5,124.3$, 124.2, 124.1, 123.9, 123.6, 122.2, 122.0, 121.8, 120.8, 120.2, 120.0, 111.5, 111.0, 81.5, 81.2, 74.0, 72.8, 14.9, 14.8, (double set of signals). Anal. Calcd for $\mathrm{C}_{23} \mathrm{H}_{19} \mathrm{~N}_{3} \mathrm{OS}_{2}: \mathrm{C}$, 66.16; H, 4.59; N, 10.06. Found: C, 66.05; H, 4.51; N, 10.10.

\section{2-(1H-1,2,3-Benzotriazol-1-yl)-2-(methylthio)-1-phenyl-1-(3-thienyl)-1-ethanol}

(3m) (mixture of two diastereomers): microcrystals from diethyl ether-hexane (50\%); mp 189-190 ${ }^{\circ} \mathrm{C} ;{ }^{1} \mathrm{H}$ NMR (DMSO- $\left.d_{6}\right): \delta 8.40(\mathrm{~d}, J=8.3 \mathrm{~Hz}, 1 \mathrm{H}), 8.34(\mathrm{~d}, J=8.5 \mathrm{~Hz}$, 1H), $7.95(\mathrm{~d}, J=8.2 \mathrm{~Hz}, 1 \mathrm{H}), 7.89-7.83(\mathrm{~m}, 4 \mathrm{H}), 7.53-7.45(\mathrm{~m}, 3 \mathrm{H}), 7.43-7.29(\mathrm{~m}, 4 \mathrm{H})$, 7.23-7.15 (m, 3H), 7.11-7.06 (m, 3H), 7.00-6.94 (m, 3H), 6.84-6.78 (m, 3H), $3.38(\mathrm{~s}$, $1 \mathrm{H}), 1.80(\mathrm{~s}, 3 \mathrm{H}), 1.72(\mathrm{~s}, 3 \mathrm{H})$, (double set of signals); ${ }^{13} \mathrm{C}$ NMR (DMSO- $\left.d_{6}\right): \delta 146.4$, $145.9,145.6,145.6,144.3,143.5,132.6,132.2,127.9,127.5,127.3,127.1,126.7,126.5$, $126.4,126.4,126.0,125.5,125.3,125.3,123.8,123.7,122.0,121.4,118.8,118.7,115.0$, 114.9, 80.3, 80.3, 75.6, 75.1, 13.9, 13.9, (double set of signals). Anal. Calcd for $\mathrm{C}_{19} \mathrm{H}_{17} \mathrm{~N}_{3} \mathrm{OS}$ : C, 62.10; H, 4.66; N, 11.43. Found: C, 61.77; H, 4.87; N, 11.23. 
( \pm )-1-(Methylthio)-1-(2-thienyl)acetone (4a): colorless oil (60\%); ${ }^{1} \mathrm{H} \mathrm{NMR}\left(\mathrm{CDCl}_{3}\right)$ : $\delta 7.29(\mathrm{dd}, J=5.1,1.2 \mathrm{~Hz}, 1 \mathrm{H}), 7.09-7.07(\mathrm{~m}, 1 \mathrm{H}), 6.99(\mathrm{dd}, J=5.1,3.6 \mathrm{~Hz}, 1 \mathrm{H}), 4.78$ (s, 1H), $2.32(\mathrm{~s}, 3 \mathrm{H}), 2.04(\mathrm{~s}, 3 \mathrm{H}) ;{ }^{13} \mathrm{C} \mathrm{NMR}\left(\mathrm{CDCl}_{3}\right): \delta 201.1,137.7,127.0,126.8,126.1$, 54.6, 26.6, 14.0. Anal. Calcd for $\mathrm{C}_{8} \mathrm{H}_{10} \mathrm{OS}_{2}$ : C, 51.58; H, 5.41; Found: C, 51.85; H, 5.60. The spectral data of this compound is identical to that reported in the literature.

( \pm )-1-(Methylthio)-1-(3-thienyl)acetone (4b): colorless oil (64\%); ${ }^{1} \mathrm{H} \mathrm{NMR}\left(\mathrm{CDCl}_{3}\right)$ :

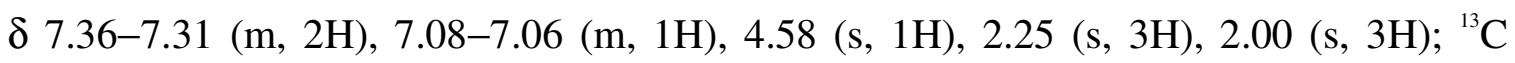
NMR $\left(\mathrm{CDCl}_{3}\right): \delta 202.3,135.3,127.3,126.2,123.6,55.4,26.6,14.0$. Anal. Calcd for $\mathrm{C}_{8} \mathrm{H}_{10} \mathrm{OS}_{2}: \mathrm{C}, 51.58 ; \mathrm{H}, 5.41$; Found: C, 51.83; H, 5.48.

( \pm )-1-(1-Benzofuran-2-yl)-1-(methylthio)acetone (4e): colorless oil $(40 \%) ;{ }^{1} \mathrm{H}$ NMR $\left(\mathrm{CDCl}_{3}\right): \delta 7.57-7.54(\mathrm{~m}, 1 \mathrm{H}), 7.46(\mathrm{~d}, J=8.2 \mathrm{~Hz}, 1 \mathrm{H}), 7.31-7.19(\mathrm{~m}, 2 \mathrm{H}), 6.87(\mathrm{~s}, 1 \mathrm{H})$, $4.66(\mathrm{~s}, 1 \mathrm{H}), 2.35(\mathrm{~s}, 3 \mathrm{H}), 2.07(\mathrm{~s}, 3 \mathrm{H}) ;{ }^{13} \mathrm{C} \mathrm{NMR}\left(\mathrm{CDCl}_{3}\right): \delta 199.6,154.8,150.7,128.0$, 124.5, 123.0, 121.0, 111.2, 106.4, 52.6, 27.1, 13.8. Anal. Calcd for $\mathrm{C}_{12} \mathrm{H}_{12} \mathrm{O}_{2} \mathrm{~S}: \mathrm{C}, 65.43$; H, 5.49; Found: C, 65.73; H, 5.50;

( \pm )-1-(1-Benzothiophen-2-yl)-1-(methylthio)acetone (4f): colorless oil $(40 \%) ;{ }^{1} \mathrm{H}$ NMR $\left(\mathrm{CDCl}_{3}\right): \delta 7.80-7.77(\mathrm{~m}, 1 \mathrm{H}), 1.74-7.70(\mathrm{~m}, 1 \mathrm{H}), 7.35-7.30(\mathrm{~m}, 3 \mathrm{H}), 4.81(\mathrm{~s}, 1 \mathrm{H})$, $2.36(\mathrm{~s}, 3 \mathrm{H}), 2.07(\mathrm{~s}, 3 \mathrm{H}) ;{ }^{13} \mathrm{C} \mathrm{NMR}\left(\mathrm{CDCl}_{3}\right): \delta 200.8,139.9,139.2,138.9,124.5,124.4$, 124.0, 123.5, 122.2, 55.3, 27.0, 14.1. Anal. Calcd for $\mathrm{C}_{12} \mathrm{H}_{12} \mathrm{OS}_{2}: \mathrm{C}, 60.98 ; \mathrm{H}, 5.12$; Found: C, 60.75; H, 5.09;

\footnotetext{
i) Tamura, Y.; Dae Choi, H.; Mizutani, M.; Ueda, Y.; Ishibashi, H. Chem. Pharm. Bull. 1982, 30, 3574 .
} 
( \pm )-1-(1-Benzothiophen-3-yl)-1-(methylthio)acetone (4g): yellow oil $\quad(71 \%) ;{ }^{1} \mathrm{H}$ NMR $\left(\mathrm{CDCl}_{3}\right): \delta 7.88-7.85(\mathrm{~m}, 1 \mathrm{H}), 7.79-7.76(\mathrm{~m}, 1 \mathrm{H}), 7.66(\mathrm{~s}, 1 \mathrm{H}), 7.40-7.37(\mathrm{~m}, 1 \mathrm{H})$, $4.81(\mathrm{~s}, 1 \mathrm{H}), 2.22(\mathrm{~s}, 3 \mathrm{H}), 2.04(\mathrm{~s}, 3 \mathrm{H}) ;{ }^{13} \mathrm{C} \mathrm{NMR}\left(\mathrm{CDCl}_{3}\right): \delta 202.0,140.2,137.4,128.9$, 125.6, 124.8, 124.4, 122.9, 121.7, 53.9, 26.4, 14.1. Anal. Calcd for $\mathrm{C}_{12} \mathrm{H}_{12} \mathrm{OS}_{2}: \mathrm{C}, 60.98$; H, 5.12; Found: C, 60.80; H, 5.09;

( \pm )-2-(Methylthio)-2-phenyl-1-(2-thienyl)-1-ethanone (4h): colorless oil $(40 \%) ;{ }^{1} \mathrm{H}$ NMR $\left(\mathrm{CDCl}_{3}\right): \delta$ 7.98-7.95 (m, 2H), 7.52-7.48 (m, 1H), 7.42-7.37 (m, 2H), 7.24-7.22 (m, 1H), 7.09-7.08 (m, 1H), 6.93-6.89 (m, 1H), $5.71(\mathrm{~d}, J=1.1 \mathrm{~Hz}, 1 \mathrm{H}), 1.99(\mathrm{~d}, J=1.6$ $\mathrm{Hz}, 3 \mathrm{H}) ;{ }^{13} \mathrm{C} \mathrm{NMR}\left(\mathrm{CDCl}_{3}\right): \delta 192.5,138.3,135.2,133.4,128.8,128.7,127.3,126.5$, 126.4, 47.9, 13.6. Anal. Calcd for $\mathrm{C}_{13} \mathrm{H}_{12} \mathrm{OS}_{2}$ : C, 62.87; H, 4.87; Found: C, 62.94; H, 5.06. ( \pm )-1-(2-Furyl)-2-(methylthio)-2-phenyl-1-ethanone (4i): yellow oil (23\%); ${ }^{1} \mathrm{H}$ NMR $\left(\mathrm{CDCl}_{3}\right): \delta 8.02-7.99(\mathrm{~m}, 2 \mathrm{H}), 7.60-7.55(\mathrm{~m}, 1 \mathrm{H}), 7.49-7.42(\mathrm{~m}, 3 \mathrm{H}), 6.59-6.57(\mathrm{~m}, 1 \mathrm{H})$, 6.38-6.36 (m, 1H), $5.57(\mathrm{~s}, 1 \mathrm{H}), 2.05(\mathrm{~s}, 3 \mathrm{H}) ;{ }^{13} \mathrm{C} \mathrm{NMR}\left(\mathrm{CDCl}_{3}\right): \delta 191.2,148.3,142.6$, 135.3, 133.4, 128.7, 110.7, 110.0, 46.1, 13.2. Anal. Calcd for $\mathrm{C}_{13} \mathrm{H}_{12} \mathrm{O}_{2} \mathrm{~S}: \mathrm{C}, 67.22 ; \mathrm{H}$, 5.21; Found: C, 67.10; H, 5.39;

( \pm )-1-(1-Benzofuran-2-yl)-2-(methylthio)-2-phenyl-1-ethanone $\quad(\mathbf{4 k})$ : yellow oil (46\%); ${ }^{1} \mathrm{H}$ NMR $\left(\mathrm{CDCl}_{3}\right): \delta$ 7.97-7.95 (m, 2H), 7.49-7.45 (m, 2H), 7.40-7.35 (m, 3H), 7.22-7.10 (m, 2H), $6.92(\mathrm{~s}, 1 \mathrm{H}), 5.59(\mathrm{~s}, 1 \mathrm{H}), 2.00(\mathrm{~s}, 3 \mathrm{H}) ;{ }^{13} \mathrm{C} \mathrm{NMR}\left(\mathrm{CDCl}_{3}\right): \delta 190.8$, $154.8,151.1,135.1,133.5,128.7,128.7,128.2,124.4,122.9,121.1,111.2,107.0,46.1$, 13.3. Anal. Calcd for $\mathrm{C}_{17} \mathrm{H}_{14} \mathrm{O}_{2} \mathrm{~S}$ : C, 72.31; H, 5.00; Found: C, 72.27; H, 5.30.

( \pm )-1-(1-Benzothiophen-2-yl)-2-(methylthio)-2-phenyl-1-ethanone $\quad$ (4l): yellow microcrystals from diethyl ether- hexane to give (40\%); mp 71-72 ${ }^{\circ} \mathrm{C} ;{ }^{1} \mathrm{H} \mathrm{NMR}\left(\mathrm{CDCl}_{3}\right)$ : 
$\delta 8.06(\mathrm{~d}, J=7.3 \mathrm{~Hz}, 2 \mathrm{H}), 7.80-7.77(\mathrm{~m}, 1 \mathrm{H}), 7.72-7.69(\mathrm{~m}, 1 \mathrm{H}), 7.59-7.54(\mathrm{~m}, 1 \mathrm{H})$, 7.49-7.45 (m, 2H), $7.40(\mathrm{~s}, 1 \mathrm{H}), 7.34-7.26(\mathrm{~m}, 2 \mathrm{H}), 5.80(\mathrm{~s}, 1 \mathrm{H}), 2.11(\mathrm{~s}, 3 \mathrm{H}),{ }^{13} \mathrm{C}$ NMR $\left(\mathrm{CDCl}_{3}\right): \delta 192.4,140.2,139.6,139.2,135.2,133.5,128.8,128.7,124.4,124.3,124.3$ 123.6, 122.2, 48.7, 13.7. Anal. Calcd for $\mathrm{C}_{17} \mathrm{H}_{14} \mathrm{OS}_{2}$ : C, 68.42; H, 4.73; Found: C, 68.17; H, 4.72 .

( \pm )-2-(Methylthio)-2-phenyl-1-(3-thienyl)-1-ethanone (4m): colorless oil (71\%); ${ }^{1} \mathrm{H}$ NMR $\delta 8.00(\mathrm{~d}, J=8.2 \mathrm{~Hz}, 2 \mathrm{H}), 7.57-7.52(\mathrm{~m}, 1 \mathrm{H}), 7.47-7.42(\mathrm{~m}, 3 \mathrm{H}), 7.32-7.30(\mathrm{~m}$, 1H), $7.18(\mathrm{~d}, J=5.1 \mathrm{~Hz}, 1 \mathrm{H}), 5.57(\mathrm{~s}, 1 \mathrm{H}), 2.01(\mathrm{~s}, 3 \mathrm{H}) ;{ }^{13} \mathrm{C}$ NMR $\delta 193.7,135.8,135.7$, 133.2, 128.7, 128.6, 127.9, 126.0, 124.0, 48.8, 13.6. Anal. Calcd for $\mathrm{C}_{13} \mathrm{H}_{12} \mathrm{OS}_{2}: \mathrm{C}, 62.87$; H, 4.87; Found: C, 62.79; H, 4.87. 